\title{
Frequently-Used Properties of the Floor Function
}

\author{
Xingbo Wang1, 2* \\ 1 Department of Mechatronic Engineering, Foshan University, Foshan, China. \\ ${ }^{2}$ State Key Laboratory of Information Security, Institute of Information Engineering, Chinese Academy of \\ Sciences, Beijing 100093, China. \\ *Corresponding author. Tel.: +86075782988845; email: xbwang@fosu.edu.cn, 153668@qq.com \\ Manuscript submitted March 15, 2020; accepted June 8, 2020. \\ doi: 10.17706/ijapm.2020.10.4.135-142
}

\begin{abstract}
The paper collects 42 frequently-used properties of the floor function, including 35 ones from other literatures and 7 newly added-and-proved ones. The collected properties cover basic inequalities, basic identities, conditional inequalities, conditional equalities and practical formulas. The paper is helpful for scholars of mathematics and computer science and technology in reading and writing scientific works, reasoning and designing algorithms.
\end{abstract}

Key words: Computational science, floor function, formula, mathematical reasoning.

\section{Introduction}

The floor function, which is also called the greatest integer function (see in [1]), is a function that takes an integer value. For arbitrary real number $x$, the floor function of $x$, denoted by $\lfloor x\rfloor$, is defined by an inequality of $x-1<\lfloor x\rfloor \leq x$ or equivalently $\lfloor x\rfloor \leq x<\lfloor x\rfloor+1$. The floor function frequently occurs in many aspects of mathematics and computer science. However, as I stated in article [2], except the Graham's book [3], it is hard to find another book or a literature that introduces in general the know-of of the function although people can find something via the Internet, e.g., the wikipedia [4]. Since Graham's book was first published 30 year's ago and its following-up editions made few modifications on the part of the floor function, it is necessary to sort out the properties of the function as a reference for researchers.

In 2017 and 2019, I proved respectively several formulas for the function and made brief summaries on the frequently-used properties by my work together with certain formulas collected from previous literatures, as seen in [5] and [6]. In the past two years, I proved several new results and thus I put them together with the 2019 summary to form this literature.

\section{Definition and Notation}

The floor function of real number $x$ is denoted by symbol $\lfloor x\rfloor$ that satisfies $\lfloor x\rfloor \leq x<\lfloor x\rfloor+1$; the fraction part of $\mathrm{x}$ is denoted by symbol $\{x\}$ that satisfies $x=\lfloor x\rfloor+\{x\}$; the ceiling function of $x$ is denoted by symbol $\lceil x\rceil$ that fits $x \leq\lceil x\rceil<x+1$. In this whole article, $A \Rightarrow B$ means conclusion $B$ can be derived from condition $A$; $A \Leftrightarrow B$ means $B$ holds if and only if $A$ holds. Symbol $\boldsymbol{Z}$ means the integer set, $x \in Z$ means $x$ is an integer and $x \notin Z$ indicates $x$ is not an integer.

\section{Frequently Used Properties of the Floor Function}

The following properties of the floor functions are sorted by basic inequalities, conditional inequalities and 
basic equalities.

\subsection{Basic Inequalities}

In the following inequalities, $x$ and $y$ are real numbers by default.

(P1) [1] $\lfloor x\rfloor+\lfloor y\rfloor \leq\lfloor x+y\rfloor \leq\lfloor x\rfloor+\lfloor y\rfloor+1$

(P2) [7] $\lfloor x\rfloor-\lfloor y\rfloor-1 \leq\lfloor x-y\rfloor \leq\lfloor x\rfloor-\lfloor y\rfloor<\lfloor x\rfloor-\lfloor y\rfloor+1$

(P3) [1], [3] $\lfloor 2 x\rfloor+\lfloor 2 y\rfloor \geq\lfloor x\rfloor+\lfloor y\rfloor+\lfloor x+y\rfloor$

(P4) [7] $\lfloor(m+n) x\rfloor+\lfloor(m+n) y\rfloor \geq\lfloor m x\rfloor+\lfloor m y\rfloor+\lfloor n x+n y\rfloor$ with $m$ and $n$ being positive integers

(P5) [7] $\lfloor n x\rfloor+\lfloor n y\rfloor \geq(n-1)\lfloor x+y\rfloor+\lfloor x\rfloor+\lfloor y\rfloor$ with $n$ being a positive integer

(P6) [1], [7] $\lfloor x y\rfloor \geq\lfloor x\rfloor\lfloor y\rfloor$ with $x, y \geq 0$.

(P7) [8] $\left\lfloor\frac{y}{x}\right\rfloor \leq \frac{\lfloor y\rfloor}{\lfloor x\rfloor}$ with $x \geq 1$ and $y>0$.

(P8) [3] $n\lfloor x\rfloor \leq\lfloor n x\rfloor ; n\lfloor x\rfloor=\lfloor n x\rfloor \Leftrightarrow n\{x\}<1$, where $n$ is a positive integer.

(P9) [9] $\left\lfloor\frac{q}{p}\right\rfloor \geq \frac{q+1}{p}-1$ for arbitrary positive integers $p$ and $q$;

\subsection{Conditional Inequalities}

In the following inequalities, $x$ and $y$ are real numbers, and $n$ is an integer.

(P10) [3] $x<n \Leftrightarrow\lfloor x\rfloor<n, n \leq x \Leftrightarrow n \leq\lfloor x\rfloor$

(P11) [3] $x<n \leq y \Leftrightarrow\lfloor x\rfloor<n \leq\lfloor y\rfloor$

(P12) [2] $\lfloor x\rfloor>\lfloor y\rfloor \Rightarrow x>y$

(P13) [2], [7] $x \leq y \Rightarrow\lfloor x\rfloor \leq\lfloor y\rfloor$

\subsection{Basic Equalities}

In the following equalities, $x$ and $y$ are real numbers, $m$ and $n$ are integers.

(P14) [3], [7] $\lfloor n+x\rfloor=n+\lfloor x\rfloor$.

(P15) [7] $\left\lfloor\frac{\lfloor x\rfloor}{m}\right\rfloor=\left\lfloor\frac{x}{m}\right\rfloor$ with $\quad m \geq 1$.

(P16) [7] $\lfloor-x\rfloor=\left\{\begin{array}{l}-\lfloor x\rfloor, x \in \boldsymbol{Z} \\ -\lfloor x\rfloor-1, x \notin \boldsymbol{Z}\end{array}\right.$

(P17) [3], [7] $\lfloor n x\rfloor=\lfloor x\rfloor+\left\lfloor x+\frac{1}{n}\right\rfloor+\ldots+\left\lfloor x+\frac{n-1}{n}\right\rfloor$ with $n>0$, particularly, $\lfloor x\rfloor+\left\lfloor x+\frac{1}{2}\right\rfloor=\lfloor 2 x\rfloor$ and $\left\lfloor\frac{x}{2}\right\rfloor+$ $\left\lfloor\frac{x+1}{2}\right\rfloor=\lfloor x\rfloor$.

(P18) [3] $\lfloor x\rfloor=\left\lfloor\frac{x}{n}\right\rfloor+\left\lfloor\frac{1+x}{n}\right\rfloor+\ldots+\left\lfloor\frac{n-1+x}{n}\right\rfloor$, particularly, $\left\lfloor\frac{x}{2}\right\rfloor+\left\lfloor\frac{x+1}{2}\right\rfloor=\lfloor x\rfloor$

(P19) [3] $\left\lceil\frac{n}{m}\right\rceil=\left\lfloor\frac{n-1}{m}\right\rfloor+1$ with $m \geq 1$.

(P20) [1], [3] $\lfloor\sqrt{x}\rfloor=\lfloor\sqrt{\lfloor x\rfloor}\rfloor$ with $x \geq 0$

(P21) [3] $\left\lfloor\log _{b} x\right\rfloor=\left\lfloor\log _{b}\lfloor x\rfloor\right\rfloor$ with $x>0$

(P22) [3] $\left\lfloor\log _{b} m\right\rfloor+1=\left\lceil\log _{b}(m+1)\right\rceil$ with $m \geq 1$.

(P23) [3] $\left\lfloor\frac{\left\lfloor\frac{a}{b}\right\rfloor}{c}\right\rfloor=\left\lfloor\frac{a}{b c}\right\rfloor$ for an arbitrary integer $a$ and positive integers $b$ and $c$. 
(P24) [1], [7] $\left\lfloor\frac{m+1}{n}\right\rfloor= \begin{cases}\left\lfloor\frac{m}{n}\right\rfloor & , n \nmid m+1 \\ \left\lfloor\frac{m}{n}\right\rfloor+1, n \mid m+1\end{cases}$

(P25) [7] $\sum_{1 \leq n \leq x} 1=\lfloor x\rfloor$

(P26) [9] $\lfloor\sqrt{n}+\sqrt{n+1}\rfloor=\lfloor\sqrt{4 n+1}\rfloor=\lfloor\sqrt{4 n+2}\rfloor=\lfloor\sqrt{4 n+3}\rfloor$

(P27) [1], [3] It needs $\left\lfloor\log _{2} N\right\rfloor+1$ binary bits to express decimal integer $N$ in its binary expression. A positive integer $n$ with base b has $\left\lfloor\log _{b} n\right\rfloor+1$ digits.

(P28) [11] Let $N$ be an integer; then $N-\lfloor\sqrt{N}\rfloor^{2} \geq 0$.

(P29) [7] Let $m$ and $p$ be positive integers; then number of $p$ 's multiples from 1 to $m$ is calculated by $\left\lfloor\frac{m}{p}\right\rfloor$.

(P30) [10] Let $m, n$ and $p$ be positive integers such that $1<p<m<n$; then number of $p$ 's multiples from $m$ to $n$ is calculated by

$$
v(m, n, p)=\left\{\begin{array}{l}
\left\lfloor\frac{n}{p}\right\rfloor-\left\lfloor\frac{m}{p}\right\rfloor, p \nmid m \\
\left\lfloor\frac{n}{p}\right\rfloor-\left\lfloor\frac{m}{p}\right\rfloor+1, p \mid m
\end{array}\right.
$$

(P31) [5] Arbitrary positive integer $i$ yields

$$
i-1 \leq 2\left\lfloor\frac{i}{2}\right\rfloor \leq i
$$

Arbitrary positive even integer $e$ yields

$$
2\left\lfloor\frac{e}{2}\right\rfloor=e
$$

And arbitrary positive old integer $o$ yields

$$
2\left\lfloor\frac{o}{2}\right\rfloor=o-1
$$

(P32) [12] Let $\alpha$ and $x$ be positive real numbers; then it holds

$$
\alpha\lfloor x\rfloor-1<\lfloor\alpha x\rfloor<\alpha(\lfloor x\rfloor+1)
$$

Particularly, if $\alpha$ is a positive integer, say $\alpha=n$, then it yields

$$
n\lfloor x\rfloor \leq\lfloor n x\rfloor \leq n(\lfloor x\rfloor+1)-1
$$

(P33) [12] For arbitrary positive real numbers $\alpha, x$ and $y$ with $x>y$, it holds

$$
\lfloor\alpha(x-y)\rfloor+\alpha\lfloor y-x\rfloor \leq 0
$$

(P34) [12]. For arbitrary odd integer $n \geq 7$, it holds

$$
1+\left\lfloor\log _{2} n\right\rfloor \leq \frac{n-1}{2}
$$

(P35) [13] For positive integer $k$ and real number $x>0$, it holds

$$
0 \geq 2^{k}\left\lfloor\frac{x}{2^{k}}\right\rfloor-\lfloor x\rfloor \geq\left\{\begin{array}{l}
1-2^{k}, 0 \leq k \leq\left\lfloor\log _{2} x\right\rfloor \\
-\lfloor x\rfloor, k>\left\lfloor\log _{2} x\right\rfloor
\end{array}\right.
$$




\section{Some New Results}

Here lists some newly found and proved equalities and inequalities.

(P36) $\lfloor\alpha\lfloor x\rfloor\rfloor \leq \min (\lfloor\alpha x\rfloor, \alpha\lfloor x\rfloor$ ) for positive numbers $\alpha$ and $x$. Particularly,

$$
\lfloor\alpha x\rfloor-1 \leq\lfloor\alpha\lfloor x\rfloor\rfloor \leq\lfloor\alpha x\rfloor
$$

When $0 \leq \alpha<1$.

Proof. See the following three steps:

1). $\lfloor\alpha\lfloor x\rfloor\rfloor \leq \alpha\lfloor x\rfloor$ by definition;

2). $\alpha\lfloor x\rfloor \leq \alpha x \Rightarrow\lfloor\alpha\lfloor x\rfloor\rfloor \leq\lfloor\alpha x\rfloor$ by (P13);

3). $\lfloor\alpha\lfloor x\rfloor\rfloor=\lfloor\alpha(x-\{x\})\rfloor=\lfloor\alpha x-\alpha\{x\})\rfloor \geq\lfloor\alpha x\rfloor-\lfloor\alpha\{x\})\rfloor-1=\lfloor\alpha x\rfloor-1$.

(P37) For an arbitrary positive integer $k$ and an arbitrary odd integer $N \geq 1$, it holds

$$
\left\lfloor\frac{N}{2^{k}}\right\rfloor=\left\lfloor\frac{N-1}{2^{k}}\right\rfloor
$$

Proof. Consider the case $k=1$ and $N=2 s+1$ with integer $s \geq 0$. It yields

$$
\left(\left\lfloor\frac{N}{2}\right\rfloor=s+\left\lfloor\frac{1}{2}\right\rfloor=s\right)=\left(\left\lfloor\frac{N-1}{2}\right\rfloor=s\right)
$$

Now assume $k>1$ and $N=2^{k} s+r$ with $s \geq 0$ being an integer and $0<r \leq 2^{k}-1$ being odd; without loss of generality, let $r=2 t+1$ with integer $0 \leq t \leq 2^{k-1}-1$; then $N-1=2^{k} s+2 t$ and $\left\lfloor\frac{N-1}{2^{k}}\right\rfloor=s+\left\lfloor\frac{t}{2^{k-1}}\right\rfloor=s$. Meanwhile, it knows

$$
\left\lfloor\frac{N}{2^{k}}\right\rfloor=s+\left\lfloor\frac{2 t+1}{2^{k}}\right\rfloor=s
$$

Because $0<r=2 t+1 \leq 2^{k}-1$.

Remark on (P37). The condition that $N$ is odd is mandatory because this property does not hold for an even integer $N$. A simple counterexample is $\left\lfloor\frac{4}{2}\right\rfloor \neq\left\lfloor\frac{4-1}{2}\right\rfloor$. Actually, when $k=1$ taking $N=2 s$ yields $\left\lfloor\frac{N}{2}\right\rfloor=s$ while $\left\lfloor\frac{N-1}{2}\right\rfloor=s+\left\lfloor-\frac{1}{2}\right\rfloor=s-1$. When $k>1$ taking $N=2^{k} s+2 t$ with $s \geq 0$ and $0 \leq t \leq 2^{k-1}-1$ being integers leads to $\left\lfloor\frac{N}{2^{k}}\right\rfloor=s+\left\lfloor\frac{t}{2^{k-1}}\right\rfloor=s$ and $\left\lfloor\frac{N-1}{2^{k}}\right\rfloor=s+\left\lfloor\frac{2 t-1}{2^{k}}\right\rfloor=s+\left\lfloor\frac{t}{2^{k-1}}-\frac{1}{2^{k}}\right\rfloor$, which results in by (P2)

$$
\left\lfloor\frac{N}{2^{k}}\right\rfloor-\left\lfloor\frac{N-1}{2^{k}}\right\rfloor=\left\{\begin{array}{l}
0, t \neq 0 \\
1, t=0
\end{array}\right.
$$

(P38) For an arbitrary positive integer $k$ and even integer $N \geq 2$, it holds

$$
\left\lfloor\frac{N}{2^{k}}\right\rfloor=\left\lfloor\frac{N+1}{2^{k}}\right\rfloor
$$

Proof. Consider the case $k=1$ and $N=2 s$ with integer $s \geq 0$. Then

$$
\left(\left\lfloor\frac{N}{2}\right\rfloor=s\right)=\left(\left\lfloor\frac{N+1}{2}\right\rfloor=s+\left\lfloor\frac{1}{2}\right\rfloor=s\right)
$$

Now assume $k>1$ and $N+1=2^{k} s+r=2^{k} s+2 t+1$ with integers $s \geq 0$ and $0 \leq t \leq 2^{k-1}-1$; then 


$$
\left\lfloor\frac{N+1}{2^{k}}\right\rfloor=s+\left\lfloor\frac{r}{2^{k}}\right\rfloor=s
$$

Because $0<r=2 t+1 \leq 2^{k}-1$.

Meanwhile by $N=2^{k} s+2 t$ with $0 \leq t \leq 2^{k-1}-1$, it holds

$$
\left\lfloor\frac{N}{2^{k}}\right\rfloor=s+\left\lfloor\frac{t}{2^{k-1}}\right\rfloor=s
$$

Thus it knows $\left\lfloor\frac{N}{2^{k}}\right\rfloor=\left\lfloor\frac{N+1}{2^{k}}\right\rfloor$ when $N$ is even.

Remark on (P38). The condition that $N$ is even is mandatory because this property does not hold for an odd integer $N$. A simple counterexample is $\left\lfloor\frac{3+1}{2}\right\rfloor \neq\left\lfloor\frac{3}{2}\right\rfloor$. Readers can confirm the general cases by referring to the Remark on (P37).

(P39) For arbitrary positive real numbers $x$ and $y$ satisfying $x \geq y$, it holds

$$
\lfloor x-y\rfloor=0 \Rightarrow\lfloor y\rfloor \leq\lfloor x\rfloor \leq\lfloor y\rfloor+1
$$

Proof. First is to prove the necessity as following reasoning:

$$
\begin{aligned}
& \lfloor x-y\rfloor=0 \Rightarrow\lfloor\lfloor x\rfloor+\{x\}-\lfloor y\rfloor-\{y\}\rfloor=0 \\
& \Rightarrow\lfloor x\rfloor-\lfloor y\rfloor+\lfloor\{x\}-\{y\}\rfloor=0
\end{aligned}
$$

Since $\{x\}$ and $\{y\}$ are positive real numbers satisfying $0 \leq\{x\}<1$ and $0 \leq\{y\}<1$, it knows $-1<$ $\{x\}-\{y\}<1 \Rightarrow-1 \leq\lfloor\{x\}-\{y\}\rfloor \leq 0$ and thus

$$
\begin{aligned}
& \lfloor x\rfloor-\lfloor y\rfloor+\lfloor\{x\}-\{y\}\rfloor=0 \\
& \Rightarrow\lfloor y\rfloor \leq\lfloor x\rfloor \leq\lfloor y\rfloor+1
\end{aligned}
$$

(P40) For positive integer $\alpha$ and $0 \leq x<1$, it holds

$$
\left\lfloor\log _{2}\left(2^{\alpha}-1+x\right)\right\rfloor=\alpha-1 \text { and }\left\lfloor\log _{2}\left(2^{\alpha}+x\right)\right\rfloor=\alpha
$$

Proof. By property of $\mathrm{T}_{3}$ tree, $2^{\alpha}-1$ is on level $\alpha-2$ of $\mathrm{T}_{3}$ (see in [14]). That is

$$
\alpha-2=\left\lfloor\log _{2}\left(2^{\alpha}-1\right)\right\rfloor-1 \Rightarrow\left\lfloor\log _{2}\left(2^{\alpha}-1\right)\right\rfloor=\alpha-1
$$

Since $\log _{2}\left(2^{\alpha}-1\right)<\log _{2}\left(2^{\alpha}-1+x\right)<\log _{2}\left(2^{\alpha}\right)=\alpha$, by (P13) it holds

$$
\alpha-1=\left\lfloor\log _{2}\left(2^{\alpha}-1\right)\right\rfloor \leq\left\lfloor\log _{2}\left(2^{\alpha}-1+x\right)\right\rfloor \leq \log _{2}\left(2^{\alpha}-1+x\right)<\alpha
$$

Likewise, $2^{\alpha}+1$ is on level $\alpha-1$ of $\mathrm{T}_{3}$, namely

$$
\left\lfloor\log _{2}\left(2^{\alpha}+1\right)\right\rfloor-1=\alpha-1 \Rightarrow\left\lfloor\log _{2}\left(2^{\alpha}+1\right)\right\rfloor=\alpha
$$

Since $\log _{2} 2^{\alpha}<\log _{2}\left(2^{\alpha}+x\right)<\log _{2}\left(2^{\alpha}+1\right)$, it knows

$$
\alpha \leq\left\lfloor\log _{2}\left(2^{\alpha}+x\right)\right\rfloor \leq\left\lfloor\log _{2}\left(2^{\alpha}+1\right)\right\rfloor=\alpha
$$

(P41). For integers $n \geq 0$ and $\alpha \geq 0$, it holds

$$
\left\lfloor\frac{-n-1}{2^{\alpha}}\right\rfloor=-1-\left\lfloor\frac{n}{2^{\alpha}}\right\rfloor
$$

Proof. By (P16), the proof considers two cases: $n+1$ is divisible by $2^{\alpha}$ and it is not. For the case $n+1$ is 
divisible by $2^{\alpha}$, let $n+1=2^{\alpha} s$ with $s \geq 1$ being an integer; then

$$
n+1 \equiv 0 \bmod 2^{\alpha} \Rightarrow \frac{n+1}{2^{\alpha}}=s \Rightarrow\left\lfloor\frac{-n-1}{2^{\alpha}}\right\rfloor=-s
$$

And this time

$$
n=2^{\alpha} s-1 \Rightarrow\left\lfloor\frac{n}{2^{\alpha}}\right\rfloor=\left\lfloor s-\frac{1}{2^{\alpha}}\right\rfloor=s-1
$$

Consequently, it holds

$$
\left\lfloor\frac{-n-1}{2^{\alpha}}\right\rfloor=-1-\left\lfloor\frac{n}{2^{\alpha}}\right\rfloor
$$

For the case $n+1$ is not divisible by $2^{\alpha}$, let $n+1=2^{\alpha} s+r$; then integer $s \geq 1$ and

$$
0<r \leq 2^{\alpha}-1 \Rightarrow 0 \leq r-1 \leq 2^{\alpha}-2
$$

Accordingly, it holds

$$
\begin{aligned}
& n+1=2^{\alpha} s+r, 0<r \leq 2^{\alpha}-1 \\
& \Rightarrow n=2^{\alpha} s+r-1 \\
& \Rightarrow\left\lfloor\frac{n+1}{2^{\alpha}}\right\rfloor=s=\left\lfloor\frac{n}{2^{\alpha}}\right\rfloor
\end{aligned}
$$

And consequently

$$
\left\lfloor\frac{-n-1}{2^{\alpha}}\right\rfloor=-1-\left\lfloor\frac{n}{2^{\alpha}}\right\rfloor
$$

(P42). Given positive integers $k$ and $N$; let $m=\left\lfloor\log _{2} N\right\rfloor-k>0$. Then there must be an odd one among $\left\lfloor\frac{N}{2^{k+1}}\right\rfloor,\left\lfloor\frac{N}{2^{k+2}}\right\rfloor, \ldots$, and $\left\lfloor\frac{N}{2^{k+m}}\right\rfloor$ if $\left\lfloor\frac{N}{2^{k}}\right\rfloor>0$ is even. Similarly, there must be an even one among $\left\lfloor\frac{N}{2^{k+1}}\right\rfloor,\left\lfloor\frac{N}{2^{k+2}}\right\rfloor, \ldots$, and $\left\lfloor\frac{N}{2^{k+m}}\right\rfloor$ if $\left\lfloor\frac{N}{2^{k}}\right\rfloor>0$ is odd.

Proof. Consider the case $\left\lfloor\frac{N}{2^{k}}\right\rfloor$ is even. Suppose $\left\lfloor\frac{N}{2^{k}}\right\rfloor=2^{\alpha} s$ with $\quad \alpha \geq 1$ being a positive integer and $s \geq 1$ being an odd integer; then

$$
N=2^{k}\left\lfloor\frac{N}{2^{k}}\right\rfloor+r=2^{k+\alpha} s+r, 0 \leq r \leq 2^{k}-1
$$

This follows

$$
\left\lfloor\frac{N}{2^{k+\alpha}}\right\rfloor=s
$$

Which is odd.

Now check the bound of $\alpha$. Since $N \geq 2^{k+\alpha} s$, it knows

$$
\begin{aligned}
& 2^{k+\alpha} \leq \frac{N}{s} \leq N \\
& \Rightarrow \alpha \leq \log _{2} N-k \\
& \Rightarrow \alpha \leq\left\lfloor\log _{2} N\right\rfloor-k
\end{aligned}
$$

For the case $\left\lfloor\frac{N}{2^{k}}\right\rfloor$ is odd, suppose $\left\lfloor\frac{N}{2^{k}}\right\rfloor=2^{\alpha} s+1$ with $\alpha \geq 1$ being a positive integer and $s \geq 1$ being an 
odd integer;

$$
N=2^{k}\left\lfloor\frac{N}{2^{k}}\right\rfloor+r=2^{k+\alpha} s+2^{k}+r, 0 \leq r \leq 2^{k}-1
$$

and for a positive integer $\mathrm{x}$, it holds

$$
\left\lfloor\frac{N}{2^{k+x}}\right\rfloor=2^{\alpha-x} s+\left\lfloor\frac{2^{k}+r}{2^{k+x}}\right\rfloor
$$

Since $2^{k} \leq 2^{k}+r \leq 2^{k+1}-1<2^{k+1}$, it knows $\left\lfloor\frac{2^{k}+r}{2^{k+x}}\right\rfloor=0$ when $x \geq 1$. Consequently, if $1 \leq x \leq \alpha-1$ then $\left\lfloor\frac{N}{2^{k+x}}\right\rfloor=2^{\alpha-x} s$ is even. Considering $\quad N=2^{k}\left\lfloor\frac{N}{2^{k}}\right\rfloor+r=2^{k+\alpha} s+2^{k}+r>2^{k+\alpha}$, it follows $\alpha \leq\left\lfloor\log _{2} N\right\rfloor-k$.

\section{Motivation of This Paper}

In writing a paper related with mathematics, computer science, physics and so on, mathematical reasoning plays a major role in the whole procedure. During a reasoning procedure, some minor evidences such as one or more formulas are often required to keep the reasoning correct. Since our primary middle school we have remembered tens of identities, inequalities, theorems and axioms in our minds. The things we have remembered do help us to write an excellent paper of science and technology. However, it is not so fortunate for researchers who research the number theory, the graph theory and the related subjects because they often have to face the floor function, which frequently occurs in the reasoning but does not have many citable formulas. As I mentioned in [15], the mathematical reasoning or modeling involved with the floor function always requires quite a lot of special skills related with inequalities together with discrete mathematics and it is of quite individuality because the function is defined with an inequality, $\lfloor x\rfloor \leq x<\lfloor x\rfloor+1$, and applied in occasions with integers and other discrete traits. For this reason, I have paid attention to the function and collected its formulas in my teaching and researching work. In 2017, I collected 31 properties and published them in [5]. Among the 31 properties, I proved 5 ones. They are (P12), (P13), (P28), (P30), and (P31). In 2018, I collected 35 properties among which I proved 4 new ones and published them in [6]. In this paper, I collected 42 properties among which the newly added 7 ones are proved in previous subsection. I am sure these collected properties are helpful for certain people to cite. At least I myself frequently look over them when I was writing a paper related with the issue.

The motivation that I say so many words here is to show something on how to agitate interest in scientific research. An old thing like the floor function might contain a lot of new work and any new work cannot be lack of some old things. I hope the background and history of this paper are educationally meaningful.

\section{Conflict of Interest}

The author declares that there is no conflict of interests regarding the publication of this article.

\section{Author Contributions}

Professor Xingbo Wang contributes the whole research work of the paper.

\section{Acknowledgment}

The research is supported by the Open Project Program of the State Key Lab of CAD\&CG (Grant No. A2002) and by Foshan University and Foshan Bureau of Science and Technology under project that constructs Guangdong Engineering Center of Information Security for Intelligent Manufacturing System. 


\section{References}

[1] Rosen, K. H. (2011). Elementary Number Theory and Its Applications (6th ed.). New York: Addison-Wesley.

[2] Wang, X. (2012). A mean-value formula for the floor function on integers. Mathproblems Journal, 2(4), 136-143.

[3] Graham, R. L., Knuth, D. E., \& Patashnik, O. (1994). Integer Functions. Ch.3 in Concrete Mathematics: A Foundation for Computer Science (2nd ed.). Reading, MA: Addison-Wesley.

[4] Wikipedia. (2020). Floor and ceiling functions. Retrieved from https://en.wikipedia.org/wiki/Floor_and_ceiling_functions

[5] Wang, X. (2017). Brief summary of frequently-used properties of the floor function. IOSR Journal of Mathematics, 13(5), 46-48.

[6] Wang, X. (2019). Brief summary of frequently-used properties of the floor function: Updated 2018. IOSR Journal of Mathematics, 15(1), 30-33.

[7] Pan, C. D., \& Pan, C. B. (2013). Elementary Number Theory (3rd ed.). Press of Peking University.

[8] Kuang, J. (2010). Applied Inequalities. Shandong Science and Technology Press.

[9] Liu, P. J. (2010). The Collection of Difficult Problems of Elementary Number Theory. Press of Harbin University.

[10] Wang, X. (2017). Some more new properties of consecutive odd numbers. Journal of Mathematical Research, 9(5), 47-59.

[11] Wang, X. (2017). Strategy for algorithm design in factoring RSA numbers. IOSR Journal of Computer Engineering (IOSR-JCE), 19(3), 1-7.

[12] Wang, X. (2018). Some new inequalities with proofs and comments on applications. Journal of Mathematics Research, 11(3), 15-19.

[13] Wang, X. (2018). Some inequalities on T3 tree. Advances in Pure Mathematics, 8(8), 711-719.

[14] Wang, X. (2018). T3 tree and its traits in understanding integers. Advances in Pure Mathematics, 8(5), 494-507.

[15] Wang, X. (2018). Difference property of an integer function. International Journal of Mathematics Trends and Technology, 55(3), 23-29.

Copyright (c) 2020 by the authors. This is an open access article distributed under the Creative Commons Attribution License which permits unrestricted use, distribution, and reproduction in any medium, provided the original work is properly cited (CC BY 4.0).

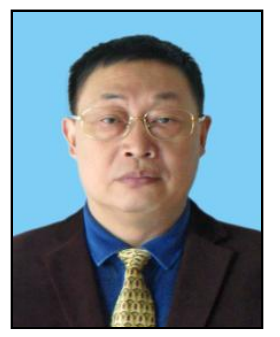

Xingbo Wang was born in Hubei, China. He got his master and doctor's degrees at National University of Defense Technology of China and had been a staff in charge of researching and developing CAD/CAM/NC technologies in the university. Since 2010, he has been a professor in Foshan University with research interests in computer application and information security. He is now the chief of Guangdong engineering center of information security for intelligent manufacturing system. Prof. Wang was in charge of more than 40 projects including projects from the National Science Foundation Committee, published 8 books and over 90 papers related with mathematics, computer science and mechatronics engineering, and invented 30 more patents in the related fields. 